\title{
PRIMARY-LIKE SUBMODULES AND A SCHEME OVER THE PRIMARY-LIKE SPECTRUM OF MODULES
}

\author{
H. FAZAELI MOGHIMI AND F. RASHEDI
}

Received 25 April, 2014

\begin{abstract}
Let $R$ be a commutative ring with identity and $M$ be a unitary $R$-module. In this paper, we obtain a scheme $\left(\mathcal{X}(M), \mathbb{O}_{\mathcal{X}(M)}\right)$ over the primary-like spectrum $\mathcal{X}(M)$ of $M$ and prove that $\left(\mathcal{X}(M), \mathbb{O}_{\mathcal{X}(M)}\right)$ is a Noetherian scheme when $R$ is a Noetherian ring.
\end{abstract}

2010 Mathematics Subject Classification: 13C99; 14A15

Keywords: Zariski topology, sheaf of rings, scheme

\section{INTRODUCTION}

Throughout this paper, all rings are commutative with identity and all modules are unital. For a submodule $N$ of an $R$-module $M,(N: M)$ denotes the ideal $\{r \in R \mid r M \subseteq N\}$ and the annihilator of $M$, denoted by $\operatorname{Ann}(M)$, is the ideal $(0: M)$. An $R$-module $M$ is called faithful if $\operatorname{Ann}(M)=(0)$. A submodule $P$ of an $R$-module $M$ is said to be $p$-prime if $P \neq M$ and for $p=(P: M)$, whenever $r m \in P$ (where $r \in R$ and $m \in M$ ) then $m \in P$ or $r \in p$ [7,11]. The collection of all prime submodules of $M$ is denoted by $\operatorname{Spec}(M)$. If $N$ is a submodule of $M$, then the radical of $N$, denoted by $\operatorname{rad} N$, is the intersection of all prime submodules of $M$ containing $N$, unless no such primes exist, in which case $\operatorname{rad} N=M$ [8].

A submodule $Q$ of $M$ is said to be primary-like if $Q \neq M$ and whenever $r m \in Q$ (where $r \in R$ and $m \in M)$ implies $r \in(Q: M)$ or $m \in \operatorname{rad} Q$ [4]. An $R$-module $M$ is said to be primeful or $\psi$-module if either $M=(0)$ or $M \neq(0)$ and the map $\psi: \operatorname{Spec}(M) \rightarrow \operatorname{Spec}(R / \operatorname{Ann}(M))$, defined by $\psi(P)=(P: M) / \operatorname{Ann}(M)$ is surjective [10]. If $M / N$ is a $\psi$-module over $R$, then $\sqrt{(N: M)}=(\operatorname{rad} N: M)$ [10, Proposition 5.3]. It is easily seen that, if $Q$ is a primary-like submodule of $M$ such that $M / Q$ is a $\psi$-module over $R$, then $(Q: M)$ is a primary ideal of $R$ and so $p=\sqrt{(Q: M)}$ is a prime ideal of $R$ [4, Lemma 2.1], and in this case $Q$ is called a $p$-primary-like submodule of $M$. The primary-like spectrum of $M$ denoted by $\chi(M)$ is defined to be the set of all primary-like submodules $Q$ of $M$, where $M / Q$ is a $\psi$-module.

An $R$-module $M$ is said to be a $\phi$-module if either $M=(0)$ or $M \neq(0)$ and the 
$\operatorname{map} \phi: \mathcal{X}(M) \rightarrow \operatorname{Spec}(R / \operatorname{Ann}(M))$ defined by $\phi(Q)=\sqrt{(Q: M)} / \operatorname{Ann}(M)$ is surjective. If $M$ is a $\phi$-module and $p$ is a prime ideal of $R$ containing $A n n(M)$, then there exists $Q \in \mathcal{X}(M)$ such that $\psi\left(S_{p}(Q+p M)\right)=\phi(Q)=p / A n n(M)$, where $S_{p}(Q+p M)=\{m \in M \mid c m \in Q+p M$ for some $c \in R \backslash p\}$ is the saturation of $Q+p M$ in $M$ with respect to $p$. Thus every $\phi$-module is a $\psi$-module; but the following example shows that the converse is not true.

Example 1 (cf. [10, Example 1]). Let $\Omega$ be the set of all prime integers, $M=$ $\prod_{p \in \Omega} \frac{\mathbb{Z}}{p \mathbb{Z}}$ and $M^{\prime}=\bigoplus_{p \in \Omega} \frac{\mathbb{Z}}{p \mathbb{Z}}$, where $p$ runs through $\Omega$. Hence $M$ is a faithful $\psi$-module over $\mathbb{Z}$ and $\operatorname{Spec}(M)=\left\{M^{\prime}=S_{0}(0)\right\} \cup\{p M: p \in \Omega\}$. Now if $\phi$ is surjective, then there exists $N \in \mathcal{X}(M)$ such that $\phi(N)=\sqrt{(N: M)}=0$. It follows that $(N: M)=0$. Since $M / N$ is a $\psi$-module, we have $N \subseteq \bigcap_{p \in \Omega} p M=0$. But 0 is not prime and so is not primary-like because $\operatorname{rad} 0=0$. Hence $N \notin \mathcal{X}(M)$, a contradiction. Thus $M$ is not a $\phi$-module.

The Zariski topology on the spectrum of prime ideals of a commutative ring is one of the main tools in algebraic geometry. Recall that the spectrum $\operatorname{Spec}(R)$ of a ring $R$ consists of all prime ideals of $R$ and is non-empty. For each ideal $I$ of $R$, we set $V(I)\left(\right.$ or $\left.\left.V^{R}(I)\right)=\{p \in \operatorname{Spec}(R) \mid p \supseteq I\}\right)$. Then the sets $V(I)$, where $I$ is an ideal of $R$, satisfy the axioms for the closed sets of a topology on $\operatorname{Spec}(R)$, called the Zariski topology (for example see [3]). It is well-known that for any ring $R$, there is a sheaf of rings on $\operatorname{Spec}(R)$, called the structure sheaf, denoted by $\mathcal{O}_{\operatorname{Spec}(R)}$, defined as follows: for each prime ideal $p$ of $R$, let $R_{p}$ be the localization of $R$ at $p$. For an open set $U \subseteq \operatorname{Spec}(R)$ with respect to the Zariski topology, we define $\mathcal{O}_{\operatorname{Spec}(R)}(U)$ to be the set of functions $r: U \rightarrow \bigsqcup_{p \in U} R_{p}$, such that $r(p) \in R_{p}$, for each $p \in U$, and such that $r$ is a quotient of elements of $R$ locally: to be precise, we require that for each $p \in U$, there is a neighborhood $V$ of $p$, contained in $U$, and there are elements $a, s \in R$, such that for each $p^{\prime} \in V, s \notin p^{\prime}$ and $r\left(p^{\prime}\right)=\frac{a}{s}$ in $R_{p^{\prime}}$ (see for example [5], for definition and basic properties of the sheaf $\left.\mathcal{O}_{\operatorname{Spec}(R)}\right)$.

In the literature, there are many different generalizations of the Zariski topology for modules over commutative rings. For example, Lu has introduced a Zariski topology on $\operatorname{Spec}(M)$ whose closed sets are $V(N)=\{P \in \operatorname{Spec}(M) \mid(N: M) \subseteq(P$ : $M)\}$ for any submodule $N$ of $M$ [9]. This topological space has been investigated from several point of views (see for example [1,2, 6, 12]).

As a new generalization of the Zariski topology, the Zariski topology $\mathcal{T}$ on $\mathcal{X}(M)$ is a topology in which closed sets are of the form $v(N)=\{Q \in \mathcal{X}(M) \mid \sqrt{(N: M)} \subseteq$ $\sqrt{(Q: M)}\}$ (Lemma 1). There are various generalizations of sheaves from rings to modules in which the sheaf on $\operatorname{Spec}(M)$ is the set of all functions $r: \operatorname{Spec}(M) \rightarrow$ $\bigsqcup_{p \in U} M_{p}$ with the property similar to that for $\operatorname{Spec}(R)$ (some of these types of sheaves have been given and studied in $[6,12])$. In parallel, we introduce a sheaf $\mathbb{O}_{X(M)}$ over $\mathcal{X}(M)$.

We show that the set $\mathscr{B}=\left\{\mathcal{X}_{r}: r \in R\right\}$, where $\mathcal{X}_{r}=\mathcal{X}(M)-v(r M)$ is a basis 
for the Zariski topology over $\mathcal{X}(M)$ (Lemma 5). In particular, if $M$ is a $\phi$-module, then the elements $\mathcal{X}_{r}$ of $\mathscr{B}$ are quasi-compact (Corollary 3$)$. This basis is used to show that $\mathbb{O}_{X_{(M)}}\left(\mathcal{X}_{s}\right) \cong R_{s}$ for each $s \in R$, where $M$ is a faithful $\phi$-module and $R_{s}=\left\{\frac{a}{s^{n}}: a \in R, n \in \mathbb{N}\right\}$ (Theorem 4). Finally we show that if $M$ is a $\phi$-module over a Noetherian ring $R$ and $\mathcal{X}(M)$ is a $T_{0}$-space, then $\left(\mathcal{X}(M), \mathbb{O}_{\chi(M)}\right)$ is a Noetherian scheme (Theorem 5).

\section{The ZARISKI TOPOLOGY ON $\mathcal{X}(M)$}

We begin with a lemma to see that the sets $v(N)=\{Q \in \mathcal{X}(M) \mid \sqrt{(N: M)} \subseteq$ $\sqrt{(Q: M)}\}$ satisfy the axioms of closed sets for a topology.

Lemma 1. Let $M$ be an R-module. Then

(1) $v(0)=X(M)$ and $v(M)=\varnothing$.

(2) $\cap_{i \in I} v\left(N_{i}\right)=v\left(\sum_{i \in I}\left(N_{i}: M\right) M\right)$, for each family $\left\{N_{i} \mid i \in I\right\}$ of submodules of $M$.

(3) $v(N) \cup v\left(N^{\prime}\right)=v\left(N \cap N^{\prime}\right)$, for each pair $N, N^{\prime}$ of submodules of $M$.

Proof. (1) and (3) are trivial.

(2) Since $M / Q$ is a $\psi$-module, $(\operatorname{rad} Q: M)=\sqrt{(Q: M)}$ [10, Proposition 5.3]. Also it is easily verified that $((\operatorname{rad} Q: M) M: M)=(\operatorname{rad} Q: M))$. Using these facts we have the following implications.

$$
\begin{aligned}
Q \in \cap_{i \in I} v\left(N_{i}\right) & \Rightarrow \sqrt{(Q: M)} \supseteq \sum_{i \in I}\left(N_{i}: M\right) \\
& \Rightarrow \sqrt{(Q: M)} M \supseteq\left(\sum_{i \in I}\left(N_{i}: M\right)\right) M \\
& \Rightarrow(\sqrt{(Q: M)} M: M) \supseteq\left(\left(\sum_{i \in I}\left(N_{i}: M\right)\right) M: M\right) \\
& \Rightarrow((\operatorname{rad} Q: M) M: M) \supseteq\left(\left(\sum_{i \in I}\left(N_{i}: M\right)\right) M: M\right) \\
& \Rightarrow(\operatorname{rad} Q: M) \supseteq\left(\left(\sum_{i \in I}\left(N_{i}: M\right)\right) M: M\right) \\
& \Rightarrow \sqrt{(Q: M)} \supseteq \sqrt{\left(\left(\sum_{i \in I}\left(N_{i}: M\right)\right) M: M\right)} \\
& \Rightarrow Q \in v\left(\left(\sum_{i \in I}\left(N_{i}: M\right)\right) M\right) .
\end{aligned}
$$


For the reverse inclusion we have

$$
\begin{aligned}
Q \in v\left(\sum_{i \in I}\left(N_{i}: M\right) M\right) & \Rightarrow \sqrt{(Q: M)} \supseteq\left(\left(\sum_{i \in I}\left(N_{i}: M\right)\right) M: M\right) \\
& \Rightarrow \sqrt{(Q: M)} \supseteq\left(\left(N_{i}: M\right) M: M\right) \quad \forall i \in I \\
& \Rightarrow \sqrt{(Q: M)} \supseteq\left(N_{i}: M\right) \forall i \in I \\
& \Rightarrow \sqrt{(Q: M)} \supseteq \sqrt{\left(N_{i}: M\right)} \forall i \in I \\
& \Rightarrow Q \in \bigcap_{i \in I} v\left(N_{i}\right)
\end{aligned}
$$

We will use $\bar{R}$ and $X^{\bar{R}}$ to represent $R / \operatorname{Ann}(M)$ and $\operatorname{Spec}(R / A n n(M))$ respectively.

Proposition 1. Let $M$ be an R-module. Then $\phi^{-1}\left(V^{\bar{R}}(\bar{I})\right)=v(I M)$, for every ideal $I \in V(\operatorname{Ann}(M))$. Therefore the map $\phi$ is continuous with respect to the Zariski topology on $\mathcal{X}(M)$.

Proof. Suppose $Q \in \phi^{-1}\left(V^{\bar{R}}(\bar{I})\right)$. Then $\phi(Q) \in V^{\bar{R}}(\bar{I})$ and so $\sqrt{(Q: M)} \supseteq I$. Hence $\sqrt{(Q: M)} \supseteq \sqrt{(I M: M)}$. Thus $Q \in v(I M)$. The argument is reversible and so $\phi$ is continuous.

Theorem 1. Let $M$ be a $\phi$-module over a ring $R$. Then $\phi(v(N))=V^{\bar{R}}(\overline{(N: M)})$ and $\phi(X(M)-v(N))=X^{\bar{R}}-V^{\bar{R}}(\overline{(N: M)})$ for every submodule $N$ of $M$, i.e., $\phi$ is both closed and open.

Proof. As we have seen in Proposition $1, \phi^{-1}\left(V^{\bar{R}}(\bar{I})\right)=v(I M)$, for every ideal $I \in V(A n n(M))$. Hence for every submodule $N$ of $M, \phi^{-1}\left(V^{\bar{R}}(\overline{(N: M)})\right)=$ $v((N: M) M)=v(N)$. So $\phi(v(N))=\phi \circ \phi^{-1}\left(V^{\bar{R}}(\overline{(N: M)})\right)=V^{\bar{R}}(\overline{(N: M)})$ as $\phi$ is surjective. Thus

$$
\phi(X(M)-v(N))=\phi\left(\phi^{-1}\left(X^{\bar{R}}\right)-\phi^{-1}\left(V^{\bar{R}}(\overline{(N: M)})\right)\right)=X^{\bar{R}}-V^{\bar{R}}(\overline{(N: M)})
$$

Corollary 1. Let $M$ be an R-module. Then $\phi$ is a bijection if and only if $\phi$ is a homeomorphism.

Proposition 2. Let $M$ be an $R$-module and $Q, Q^{\prime} \in \mathcal{X}(M)$. Then the following statements are equivalent.

(1) If $v(Q)=v\left(Q^{\prime}\right)$, then $Q=Q^{\prime}$;

(2) For each $p \in \operatorname{Spec}(R)$, the set $\{Q \in \mathcal{X}(M): \sqrt{(Q: M)}=p\}$ is empty or a singleton set;

(3) $\phi$ is injective. 
Proof. (1) $\Rightarrow$ (2) Let $Q, Q^{\prime} \in \mathcal{X}(M)$ and $\sqrt{(Q: M)}=\sqrt{\left(Q^{\prime}: M\right)}=p$. Then $v(Q)=v\left(Q^{\prime}\right)$. Thus $Q=Q^{\prime}$ by $(1)$.

$(2) \Rightarrow$ (3) Suppose $Q, Q^{\prime} \in \mathcal{X}(M)$ and $\phi(Q)=\phi\left(Q^{\prime}\right)$. Hence $\sqrt{(Q: M)}=$ $\sqrt{\left(Q^{\prime}: M\right)}=p$. Thus $Q=Q^{\prime}$ by (2).

(3) $\Rightarrow$ (1) is clear.

Let $y$ be a subset of $\mathcal{X}(M)$ for a module $M$. We will denote the closure of $y$ in $\chi(M)$ by $\bar{y}$.

Proposition 3. Let $M$ be an $R$-module and let $y=\left\{Q_{1}, Q_{2}, \ldots, Q_{n}\right\}$ be a finite subset of $\mathcal{X}(M)$. Then $\bar{y}=v\left(Q_{1}\right) \cup \ldots \cup v\left(Q_{n}\right)$.

Proof. Clearly, $y \subseteq v\left(Q_{1}\right) \cup \ldots \cup v\left(Q_{n}\right)$. Assume that $\mathcal{F}$ is any closed subset of $\mathcal{X}(M)$ such that $\mathcal{Y} \subseteq \mathcal{F}$. Hence $\mathcal{F}=v(N)$ for the submodule $N$ of $M$. Let $Q \in v\left(Q_{1}\right) \cup \ldots \cup v\left(Q_{n}\right)$. Then there exists $i(1 \leq i \leq n)$ such that $Q \in v\left(Q_{i}\right)$ and so $\sqrt{\left(Q_{i}: M\right)} \subseteq \sqrt{(Q: M)}$. Since $Q_{i} \in \mathcal{F}, \sqrt{(N: M)} \subseteq \sqrt{\left(Q_{i}: M\right)} \subseteq \sqrt{(Q: M)}$ and hence $Q \in \mathcal{F}$. Hence $v\left(Q_{1}\right) \cup \ldots \cup v\left(Q_{n}\right) \subseteq \mathcal{F}$. Thus $\bar{y}=v\left(Q_{1}\right) \cup \ldots \cup v\left(Q_{n}\right)$.

The above proposition immediately yields that the following result.

Corollary 2. Let $M$ be an R-module. Then

(1) $\bar{Q}=v(Q)$ for every $Q \in \mathcal{X}(M)$.

(2) $Q^{\prime} \in \bar{Q}$ if and only if $\sqrt{\left(Q^{\prime}: M\right)} \supseteq \sqrt{(Q: M)}$ if and only if $v\left(Q^{\prime}\right) \subseteq v(Q)$.

Proof. By Proposition 3 is clear.

A topological space $\mathbb{X}$ is a $T_{0}$-space if and only if for any two distinct points in $\mathbb{X}$ there exists an open subset of $\mathbb{X}$ which contains one of the points but not the other. We know that, for any ring $R, \operatorname{Spec}(R)$ is always a $T_{0}$-space for the usual Zariski topology. In [9, P. 429], it has been shown that if $M$ is a vector space, then $(\operatorname{Spec}(M)$, is not a $T_{0}$-space. This example can be used again to show that $(\mathcal{X}(M), \mathcal{T})$ is not also a $T_{0}$-space. In fact $v(N)=\mathcal{X}(M)$ for every proper vector subspace $N$ of $M$ so that the Zariski topology on $\mathcal{X}(M)$ is the trivial topology even when $|\mathcal{X}(M)|>1$.

Theorem 2. Let $M$ be an R-module. Then $\mathcal{X}(M)$ is a $T_{0}$-space if and only if one of the statements (1) - (3) in Proposition 2 holds.

Proof. First suppose $\mathcal{X}(M)$ is a $T_{0}$-space. We prove the item(1) of proposition 2. For this assume $v(Q)=v\left(Q^{\prime}\right)$ and $Q \neq Q^{\prime}$. Since $\mathcal{X}(M)$ is a $T_{0}$-space, $\bar{Q} \neq \overline{Q^{\prime}}$. Thus by Corollary 2 we have $v(Q) \neq v\left(Q^{\prime}\right)$, a contradiction. Conversely, suppose that $Q \neq Q^{\prime} \in \mathcal{X}(M)$ and $v(Q) \neq v\left(Q^{\prime}\right)$. Therefore by Corollary $2, \bar{Q} \neq \overline{Q^{\prime}}$. Thus $\mathcal{X}(M)$ is a $T_{0}$-space.

For each $r \in R$, we set $\mathcal{X}_{r}=\mathcal{X}(M)-v(r M)$ and $D_{\bar{r}}=X^{\bar{R}}-V(\bar{R} \bar{r})$. It is easily seen that $\mathcal{X}_{0_{R}}=\varnothing, \mathcal{X}_{1_{R}}=\mathcal{X}(M)$. 
Lemma 2. Let $M$ be an $R$-module. Then $\phi\left(\mathcal{X}_{r}\right) \subseteq D_{\bar{r}}$; the equality holds if $M$ is a $\phi$-module.

Proof. By Proposition 1, $\phi^{-1}\left(D_{\bar{r}}\right)=\phi^{-1}\left(X^{\bar{R}}-V(\bar{R} \bar{r})\right)=\mathcal{X}(M)-v(r M)=$ $X_{r}$. The equality follows form Theorem 1 .

Lemma 3. Let $r, s \in R$. Then the following hold.

(1) $\mathcal{X}_{r s}=\mathcal{X}_{r} \cap \mathcal{X}_{s}$

(2) $\mathcal{X}_{r^{n}}=\mathcal{X}_{r}$ for all $n \in \mathbb{N}$.

(3) If $r$ is nilpotent, then $\mathcal{X}_{r}=\varnothing$.

Proof. (1) By Proposition 1, $\mathcal{X}_{r s}=\phi^{-1}\left(D_{\overline{r s}}\right)$. Hence $\mathcal{X}_{r s}=\phi^{-1}\left(D_{\bar{r}}\right) \cap \phi^{-1}\left(D_{\bar{s}}\right)$ $=\mathcal{X}_{r} \cap \mathcal{X}_{s}$.

(2) follows from (1).

(3) Since $r$ is nilpotent, $r^{n}=0$ for some $n \in \mathbb{N}$. Hence by (2), $\mathcal{X}_{r}=X_{r^{n}}=X_{0}=$ $\varnothing$.

Lemma 4. Let $r, s \in R$ and $M$ be a faithful $\phi$-module over $R$. If $\mathcal{X}_{s} \subseteq \mathcal{X}_{r}$, then $s \in \sqrt{R r}$.

Proof. Suppose $\mathcal{X}_{s} \subseteq \mathcal{X}_{r}$. Hence $\phi\left(\mathcal{X}_{s}\right) \subseteq \phi\left(\mathcal{X}_{r}\right)$. Since $M$ is a $\phi$-module, $D_{\bar{s}} \subseteq$ $D_{\bar{r}}$ by Lemma 2. Now since $M$ is faithful, $D_{s} \subseteq D_{r}$. Thus we have $s \in \sqrt{R r}$.

Lemma 5. Let $M$ be an $R$-module. Then the set $\mathscr{B}=\left\{X_{r}: r \in R\right\}$ forms a basis for the Zariski topology on $\mathcal{X}(M)$.

Proof. If $\mathcal{X}(M)=\varnothing$, then $\mathscr{B}=\varnothing$ and the proposition is trivially true. Hence we assume that $\mathcal{X}(M) \neq \varnothing$ and let $\mathcal{U}$ be any open set in $\mathcal{X}(M)$. Hence $\mathcal{U}=$ $\mathcal{X}(M)-v(I M)$ for some ideal $I$ of $R$. Note that $v(I M)=v\left(\sum_{a_{i} \in I} a_{i} M\right)=$ $v\left(\sum_{a_{i} \in I}\left(a_{i} M: M\right) M\right)=\cap_{a_{i} \in I} v\left(a_{i} M\right)$. Hence $\mathcal{U}=\mathcal{X}(M)-\cap_{a_{i} \in I} v\left(a_{i} M\right)=$ $\cup_{a_{i} \in I} \mathcal{X}_{a_{i}}$. This proves that $\mathscr{B}$ is a basis for the Zariski topology on $\mathcal{X}(M)$.

Theorem 3. Let $M$ be a $\phi$-module over a ring $R$. Then $\mathcal{X}_{r}$ and $\mathcal{X}_{r_{1}} \cap \ldots \cap \mathcal{X}_{r_{n}}$ are quasi-compact subsets of $\mathcal{X}(M)$.

Proof. For any open covering of $\mathcal{X}_{r}$, there is a family $\left\{r_{\lambda} \in R: \lambda \in \Lambda\right\}$ of elements of $R$ such that $\mathcal{X}_{r} \subseteq \cup_{\lambda \in \Lambda} \mathcal{X}_{r_{\lambda}}$ by Lemma 5. $D_{\bar{r}}=\phi\left(\mathcal{X}_{r}\right) \subseteq \bigcup_{\lambda \in \Lambda} \phi\left(\mathcal{X}_{r_{\lambda}}\right)=$ $\cup_{\lambda \in \Lambda} D_{\bar{r}_{\lambda}}$ by Proposition 2. It follows that there exists a finite subset $\Lambda^{\prime}$ of $\Lambda$ such that $D_{\bar{r}} \subseteq \cup_{\lambda \in \Lambda} D_{\bar{r}_{\lambda}}$ as $D_{\bar{r}}$ is quasi-compact, whence by Proposition 2, $\mathcal{X}_{r}=$ $\phi^{-1}\left(D_{\bar{r}}\right) \subseteq \cup_{\lambda \in \Lambda^{\prime}} \mathcal{X}_{r_{\lambda}}$. Thus $\mathcal{X}_{r}$ is quasi-compact. For the other part, it suffices to show that the intersection $\mathcal{X}_{r_{1}} \cap \mathcal{X}_{r_{2}}$ is a quasi-compact set. Let $\Omega$ be any open covering of $\mathcal{X}_{r_{1}} \cap \mathcal{X}_{r_{2}}$. Then $\Omega$ also covers each $\mathcal{X}_{r_{i}}(i=1,2)$ which is quasi-compact. Hence each $\mathcal{X}_{r_{i}}$ has a finite subcover and so $\mathcal{X}_{r_{1}} \cap \mathcal{X}_{r_{2}}$ has a finite subcover.

Corollary 3. Let $M$ be a $\phi$-module over a ring $R$. Then $\mathcal{X}(M)$ is quasi-compact and has a basis of quasi-compact open subsets. 


\section{SHEAF, LOCALLY RINGED SPACE AND SCHEME}

Let $M$ be an $R$-module. For every open subset $\mathcal{U}$ of $\mathcal{X}(M)$ we define $\mathbb{O}_{X(M)}(\mathcal{U})$ to be a subring of $\prod\left\{R_{p} \mid p=\sqrt{(Q: M)}, Q \in \mathcal{U}\right\}$, the ring of functions $r: \mathcal{U} \rightarrow$ $\bigsqcup\left\{R_{p} \mid p=\sqrt{(Q: M)}, Q \in \mathcal{U}\right\}$, where $r(Q) \in R_{p}$, for each $Q \in \mathcal{U}$ and $p=$ $\sqrt{(Q: M)}$ such that for each $Q \in U$, there is a neighborhood $\mathcal{V}$ of $Q$, contained in $U$ , and elements $s, t \in R$, such that for each $Q^{\prime} \in \mathcal{V}, t \notin p^{\prime}=\sqrt{\left(Q^{\prime}: M\right)}$, and $r\left(Q^{\prime}\right)=$ $\frac{s}{t}$ in $R_{p^{\prime}}$. It is easy to check that $\mathbb{O}_{X_{(M)}}(U)$ is a commutative ring with identity. Furthermore, for open sets $\mathcal{V} \subseteq U$ we define $\vartheta_{u, \mathcal{V}}: \mathbb{O}_{X(M)}(U) \rightarrow \mathbb{O}_{X(M)}(\mathcal{V})$ given by $\left\{r_{p}\right\}_{Q \in \mathcal{U}} \mapsto\left\{r_{p^{\prime}}^{\prime}\right\}_{Q^{\prime} \in \mathcal{V}}$, where $p=\sqrt{(Q: M)}$ and $p^{\prime}=\sqrt{\left(Q^{\prime}: M\right)}$. It is easy to check that $\mathbb{O}_{X(M)}$ is a sheaf of rings.

For any sheaf $\mathbb{O}$ on a topological space $\mathbb{X}$ and for any $x \in \mathbb{X}$, the stalk of $\mathbb{O}$ at $x$, denoted by $\mathbb{O}_{x}$, is $\mathbb{O}_{x}=\left\{m \mid\right.$ there exists a neighborhood $\mathbb{U}$ of $x$ and $r \in \mathbb{O}_{\mathbb{X}}(\mathbb{U})$ such that $m$ is the germ of $r$ at the point $x\}$. We say that $m$ is the germ of $r$ at the point $x$ if there exists a neighborhood $\mathbb{V}$ containing $x$ such that $\mathbb{V} \subseteq \mathbb{U}$ and $\vartheta u, \mathcal{V}(r)=m$. Two such pairs $<\mathbb{U}, r>$ and $<\mathbb{V}, s>$ define the same element for $m$ of $\mathbb{O}_{x}$ if and only if there is an open neighborhood $\mathbb{W}$ of $x$ with $\mathbb{W} \subseteq \mathbb{U} \cap \mathbb{V}$ such that $x \in \mathbb{W}$ $\left.r\right|_{\mathrm{W}}=\left.s\right|_{\mathrm{W}}$.

Lemma 6. Let $M$ be an $R$-module. Then for each $Q \in \mathcal{X}(M)$, the stalk $\mathbb{O}_{Q}$ of the sheaf $\mathbb{O}_{X(M)}$ is isomorphic to $R_{p}$, where $p=\sqrt{(Q: M)}$.

Proof. Assume $Q \in \mathcal{X}(M)$ and $m \in \mathbb{O}_{Q}$. Therefore there exists a neighborhood $\mathcal{U}$ of $Q$ and $r \in \mathbb{O}_{X(M)}(U)$ such that $m$ is the germ of $r$ at the point $Q$. For $p=$ $\sqrt{(Q: M)}$ we define $\mu: \mathbb{O}_{Q} \rightarrow R_{p}$ given by $m \mapsto r(Q)$. It is easy to check that $\mu$ is a well-defined homomorphism. Suppose $\mathcal{V}$ is another neighborhood of $Q$ and $s \in \mathbb{O}_{X(M)}(\mathcal{V})$ such that $m$ is the germ of $s$ at the point $Q$. Hence there is an open neighborhood $\mathcal{W}$ of $Q$ with $\mathcal{W} \subseteq \mathcal{U} \cap \mathcal{V}$ such that $\left.r\right|_{w}=\left.s\right|_{\mathfrak{w}}$. Since $Q \in \mathcal{W}$, then $r(Q)=s(Q)$. The map $\mu$ is surjective, because any element of $R_{p}$ can be represented as a quotient $\frac{a}{s}$ with $a \in R$ and $s \in R \backslash p$. Now we define $r\left(Q^{\prime}\right)=\frac{a}{s}$ in $R_{p^{\prime}}$, where $p^{\prime}=\sqrt{\left(Q^{\prime}: M\right)}$ for all $Q^{\prime} \in \mathcal{X}_{s}$. Then $r \in \mathbb{O}\left(\mathcal{X}_{s}\right)$. If $m$ is the equivalent class of $r$ in $\mathbb{O}_{Q}$, then $\mu(m)=\frac{a}{s}$. To show that $\mu$ is injective, let $\mathcal{U}$ be a neighborhood of $Q$, and let $r, r^{\prime} \in \mathbb{O}_{X(M)}(\mathcal{U})$ be elements having the same value $r(Q)=r^{\prime}(Q)$ at $Q$. By the definition of our sheaf, we may assume that $r=\frac{a}{s}$ and $r^{\prime}=\frac{a^{\prime}}{s^{\prime}}$, where $a, a^{\prime} \in R$ and $s, s^{\prime} \in R \backslash p$. Since $\frac{a}{s}$ and $\frac{a^{\prime}}{s^{\prime}}$ have the same image in $R_{p}$, it follows from the definition of localization that there is an $s^{\prime \prime} \in R \backslash p$ such that $s^{\prime \prime}\left(s^{\prime} a-s a^{\prime}\right)=0$ in $R$. Therefore $\frac{a}{s}=\frac{a^{\prime}}{s^{\prime}}$ in every local ring $R_{p^{\prime}}$ such that $s, s^{\prime}, s^{\prime \prime} \in R \backslash p^{\prime}$. But the set of such $Q^{\prime}$, where $p^{\prime}=\sqrt{\left(Q^{\prime}: M\right)}$ is the open set $\mathcal{X}_{s} \cap \mathcal{X}_{s^{\prime}} \cap \mathcal{X}_{s^{\prime \prime}}$, which contains $Q$. Hence $r=r^{\prime}$ in a whole neighborhood of $Q$, so they have the same stalk at $Q$.

A locally ringed space $\left(\mathbb{X}, \mathbb{O}_{\mathbb{X}}\right)$ is a pair consisting of a topological space $\mathbb{X}$ and a sheaf of rings $\mathbb{O}_{\mathbb{X}}$ all of whose stalks are local rings. 
Corollary 4. Let $M$ be an $R$-module. Then $\left(\mathcal{X}(M), \mathbb{O}_{X(M)}\right)$ is a locally ringed space.

Proof. Use Lemma 6.

Let $\left(\mathbb{X}, \mathbb{O}_{\mathbb{X}}\right)$ be a locally ringed space. The stalk $\mathbb{O}_{\mathbb{X}, x}$ of $\mathbb{X}$ at $x$ is said to be the local ring of $\mathbb{X}$ at $x$. A morphism of ringed spaces $\left(f, f^{\sharp}\right):\left(\mathbb{X}, \mathbb{O}_{\mathbb{X}}\right) \rightarrow\left(\mathbb{Y}, \mathbb{O}_{\mathcal{Y}}\right)$ is given by a continuous map $f: \mathbb{X} \rightarrow \mathbb{Y}$ and an $f$-map of sheaves of rings $f^{\sharp}: \mathbb{O}_{Y} \rightarrow$ $\mathbb{O}_{\mathbb{X}}$. You can think of $f^{\sharp}$ as a map $\mathbb{O}_{\mathcal{Y}} \rightarrow f_{*} \mathbb{O}_{\mathbb{X}}$, where $f_{*} \mathbb{O}_{\mathbb{X}}$ is a sheaf over $\mathbb{X}$ defined by $f_{*} \mathbb{O}_{\mathbb{X}}(\mathbb{V})=\mathbb{O}_{\mathbb{X}}\left(f^{-1}(\mathbb{V})\right)$ for any open subset $\mathbb{V} \subseteq \mathcal{Y}$. Moreover the restriction map on an inclusion of open sets of $Y$ is defined naturally. A morphism of locally ringed spaces $\left(f, f^{\sharp}\right):\left(\mathbb{X}, \mathbb{O}_{\mathbb{X}}\right) \rightarrow\left(\mathbb{Y}, \mathbb{O}_{\mathcal{Y}}\right)$ is a morphism of ringed spaces such that for all $x \in \mathbb{X}$ the induced ring map $\mathbb{O}_{Y}, f(x) \rightarrow \mathbb{O}_{\mathbb{X}, x}$ is a local ring map.

Proposition 4. Let $M$ and $M^{\prime}$ be $R$-modules and $\pi: M \rightarrow M^{\prime}$ be an isomorphism of modules. Then $\pi$ induces a morphism of locally ringed spaces $\left(f, f^{\sharp}\right)$ : $\left(\mathcal{X}\left(M^{\prime}\right), \mathbb{O}_{X\left(M^{\prime}\right)}\right) \rightarrow\left(\mathcal{X}(M), \mathbb{O}_{X(M)}\right)$.

Proof. We define $f\left(Q^{\prime}\right)=\pi^{-1}\left(Q^{\prime}\right)$ For any $Q^{\prime} \in \mathcal{X}\left(M^{\prime}\right)$. It is easily seen that $f$ is well-defined. In the following it is shown that $f^{-1}(v(N))=v\left((N: M) M^{\prime}\right)$ for any closed set $v(N)$ of $\mathcal{X}(M)$ and so $f$ is continuous.

$$
\begin{aligned}
Q^{\prime} \in f^{-1}(v(N)) & \Leftrightarrow f\left(Q^{\prime}\right) \in v(N) \\
& \Leftrightarrow \sqrt{\left(f\left(Q^{\prime}\right): M\right)} \supseteq \sqrt{(N: M)} \\
& \Leftrightarrow \sqrt{\left(f\left(Q^{\prime}\right): M\right)} \supseteq \sqrt{((N: M) M: M)} \\
& \Leftrightarrow \sqrt{\left(\pi^{-1}\left(Q^{\prime}\right): M\right)} \supseteq \sqrt{((N: M) M: M)} \\
& \Leftrightarrow\left(\operatorname{rad}\left(\pi^{-1}\left(Q^{\prime}\right)\right): M\right) M \supseteq(N: M) M \\
& \Leftrightarrow \pi^{-1}\left(\operatorname{rad} Q^{\prime}\right) \supseteq(N: M) M \\
& \Leftrightarrow \operatorname{rad} Q^{\prime} \supseteq(N: M) M^{\prime} \\
& \Leftrightarrow Q^{\prime} \in v\left((N: M) M^{\prime}\right) .
\end{aligned}
$$

Assume $\mathcal{U}$ is an open subset of $\mathcal{X}(M)$ and $r \in \mathbb{O}_{\mathcal{X}(M)}(U)$. Let $Q \in f^{-1}(\mathcal{U})$. Then $f(Q)=\pi^{-1}(Q) \in \mathcal{U}$. Assume that $\mathcal{W}$ is an open neighborhood of $\pi^{-1}(Q)$ with $\mathcal{W} \subseteq \mathcal{U}$ and $a, s \in R$ such that for each $Q^{\prime} \in \mathcal{W}, s \notin p^{\prime}=\sqrt{\left(Q^{\prime}: M\right)}$ and $r\left(Q^{\prime}\right)=\frac{a}{s}$ in $R_{p^{\prime}}$. Since $\pi^{-1}(Q) \in \mathcal{W}$, then $Q \in f^{-1}(\mathcal{W})$. Since $f$ is continuous, $f^{-1}(\mathcal{W})$ is an open subset of $\mathcal{X}\left(M^{\prime}\right)$. We show that for each $Q^{\prime \prime} \in f^{-1}(\mathcal{W})$ we have $s \notin \sqrt{\left(Q^{\prime \prime}: M^{\prime}\right)}$. Suppose, on the contrary, $s \in \sqrt{\left(Q^{\prime \prime}: M^{\prime}\right)}$ for some $Q^{\prime \prime} \in$ $f^{-1}(\mathcal{W})$. So $\pi^{-1}\left(Q^{\prime \prime}\right)=f\left(Q^{\prime \prime}\right) \in \mathcal{W}$. Since $\pi$ is an epimorphism, $\sqrt{\left(Q^{\prime \prime}: M^{\prime}\right)}=$ $\sqrt{\left(\pi^{-1}\left(Q^{\prime \prime}\right): M\right)}$. Hence $s \in \sqrt{\left(\pi^{-1}\left(Q^{\prime \prime}\right): M\right)}$, a contradiction. Therefore, we can 
define $f^{\sharp}(U): \mathbb{O}_{X(M)}(U) \rightarrow \mathbb{O}_{X\left(M^{\prime}\right)}\left(f^{-1}(U)\right.$ given by $f^{\sharp}(U)(r)=r \circ f$. Suppose $\mathcal{V} \subseteq \mathcal{U}$ and $Q \in f^{-1}(\mathcal{V})$. According to the commutativity of the following diagram:

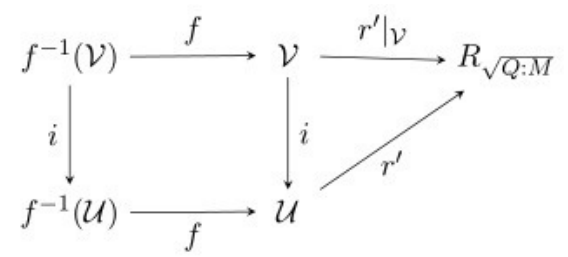

We have $\left.\left(r^{\prime} \circ f\right)\right|_{f^{-1}(\mathcal{V})}(Q)=r^{\prime} \mid \mathcal{V} \circ f(Q)$. Now, we show that the following diagram commutes.

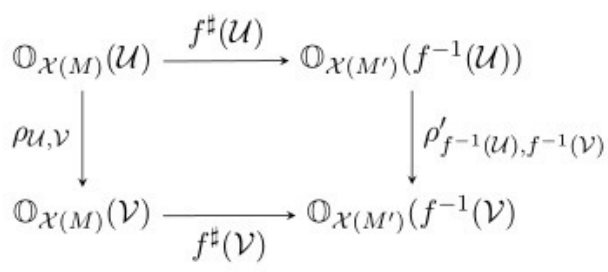

Suppose that $r^{\prime} \in \mathbb{O}_{X(M)}(\mathcal{U})$. For each $Q \in \mathcal{U}$, we have

$$
\begin{gathered}
\rho_{f^{-1}(U), f^{-1}(\mathcal{V})}^{\prime} f^{\sharp}(U)\left(r^{\prime}\right)(Q)=\rho_{f^{-1}(u), f^{-1}(\mathcal{V})}^{\prime}\left(r^{\prime} \circ f\right)(Q)= \\
\left.\left(r^{\prime} \circ f\right)\right|_{f^{-1}(\mathcal{V})}(Q)=\left.r^{\prime}\right|_{\mathcal{V}} \circ f(Q)=\rho u, \mathcal{V}\left(r^{\prime}\right) \circ f(Q)=f^{\sharp}(\mathcal{V}) \rho u, \mathcal{V}\left(r^{\prime}\right)(Q) .
\end{gathered}
$$

It follows that $f^{\sharp}: \mathbb{O}_{X(M)} \rightarrow f_{*} \mathbb{O}_{X\left(M^{\prime}\right)}$ is a morphism of sheaves. By Lemma 6, the map $f_{Q}^{\#}: \mathbb{O}_{\chi}(M), f(Q) \rightarrow \mathbb{O}_{X}\left(M^{\prime}\right), Q$ on stalks is clearly the map of local rings $R_{\sqrt{(f(Q): M)}} \rightarrow R_{\sqrt{\left(Q: M^{\prime}\right)}}$. Thus the proof is completed.

Proposition 5. Let $g: R \rightarrow R^{\prime}$ be a ring homomorphism, $M^{\prime}$ be an $R^{\prime}$-module and $M$ be a $\phi$-module over $R$ such that $\mathcal{X}(M)$ is a $T_{0}$-space and $\operatorname{Ann}_{R}(M) \subseteq$ Ann $n_{R}\left(M^{\prime}\right)$. Then $g$ induces a morphism of locally ringed spaces $\left(f, f^{\sharp}\right):\left(\mathcal{X}\left(M^{\prime}\right), \mathbb{O}_{X\left(M^{\prime}\right)}\right) \rightarrow\left(\mathcal{X}(M), \mathbb{O}_{X(M)}\right)$.

Proof. Since $A n n_{R}(M) \subseteq A n n_{R}\left(M^{\prime}\right)$, then $\bar{g}: \bar{R} \rightarrow \overline{R^{\prime}}$ is induced by $g$. It is well known $h: \operatorname{Spec}\left(R^{\prime}\right) \rightarrow \operatorname{Spec}(R)$ given by $p \mapsto g^{-1}(p)$ and $\bar{h}: X^{\overline{R^{\prime}}} \rightarrow X^{\bar{R}}$ given by $\bar{p} \mapsto \bar{g}^{-1}(\bar{p})$ are continuous maps. Also by Proposition $1, \phi_{M^{\prime}}: \mathcal{X}\left(M^{\prime}\right) \rightarrow X^{\overline{R^{\prime}}}$ is a continuous map and by Corollary 1 and Theorem $2, \phi_{M}: \mathcal{X}(M) \rightarrow X^{\bar{R}}$ is a homeomorphism. Therefore the map $f: \mathcal{X}\left(M^{\prime}\right) \rightarrow \mathcal{X}(M)$ given by $Q \mapsto \phi_{M}^{-1} \circ \bar{h} \circ$ $\phi_{M^{\prime}}(Q)$ is continuous. For each $Q \in \mathcal{X}\left(M^{\prime}\right)$, we get a local homomorphism 


$$
g_{\sqrt{\left(Q:_{R^{\prime}} M^{\prime}\right)}}: R_{h\left(\sqrt{\left(Q:_{R^{\prime}} M^{\prime}\right)}\right)} \rightarrow R^{\prime} \sqrt{\left(Q:_{R^{\prime}} M^{\prime}\right)}
$$

given by $\frac{r}{s} \mapsto \frac{g(r)}{g(s)}$. This map is well-defined, because if $s \notin h\left(\sqrt{\left(Q: R^{\prime} M^{\prime}\right)}\right)=$ $g^{-1}\left(\sqrt{\left(Q: R^{\prime} M^{\prime}\right)}\right)$, then $g(s) \notin \sqrt{\left(Q: R^{\prime} M^{\prime}\right)}$. Let $U \subseteq \mathcal{X}(M)$ be an open subset and $r \in \mathbb{O}_{X(M)}(U)$. Suppose $Q \in f^{-1}(\mathcal{U})$. Then $f(Q) \in U$ and there exists a neighborhood $\mathcal{W}$ of $f(Q)$ with $\mathcal{W} \subseteq U$ and elements $a, s \in R$ such that for each $Q^{\prime} \in \mathcal{W}$, we have $s \notin \sqrt{\left(Q^{\prime}:_{R} M\right)}$ and $r\left(Q^{\prime}\right)=\frac{a}{s} \in R_{\sqrt{\left(Q^{\prime}:_{R} M\right)}}$. Hence $s \notin \sqrt{\left(f(Q):_{R} M\right)}$. By definition of $f$, we have

$$
\begin{aligned}
f(Q) & =\left(\phi_{M}^{-1} \circ \bar{h} \circ \phi_{M^{\prime}}^{-1}\right)(Q)=\left(\phi_{M}^{-1} \circ \bar{h}\right)\left(\overline{\left(\sqrt{\left(Q: R^{\prime} M^{\prime}\right)}\right)}\right. \\
& =\phi_{M}^{-1}\left(\bar{g}^{-1}\left(\overline{\left(\sqrt{\left(Q: R^{\prime} M^{\prime}\right)}\right)}\right)=\phi_{M}^{-1} \overline{\left(g^{-1}\left(\sqrt{\left(Q: R^{\prime} M^{\prime}\right)}\right)\right.}\right) \\
& =K
\end{aligned}
$$

for some $K \in \mathcal{X}(M)$. Now since $M$ is a $\phi$-module, $\overline{\sqrt{\left(K:_{R} M\right)}}=\phi_{M}(K)=$ $\overline{g^{-1}\left(\sqrt{\left(Q:_{R^{\prime}} M^{\prime}\right)}\right)}$ and hence $\sqrt{\left(f(Q):_{R} M\right)}=\sqrt{\left(K:_{R} M\right)}=g^{-1}\left(\sqrt{\left(Q:_{R^{\prime}} M^{\prime}\right)}\right)$. Therefore $s \notin \sqrt{\left(f(Q):_{R} M\right)}$ follows that $g(s) \notin \sqrt{\left(Q:_{R^{\prime}} M^{\prime}\right)}$. Thus $g_{\sqrt{\left(Q:_{R^{\prime}} M^{\prime}\right)}}\left(\frac{a}{s}\right)$ define a section on $\mathbb{O}_{X\left(M^{\prime}\right)}\left(f^{-1}(\mathcal{W})\right)$. Since

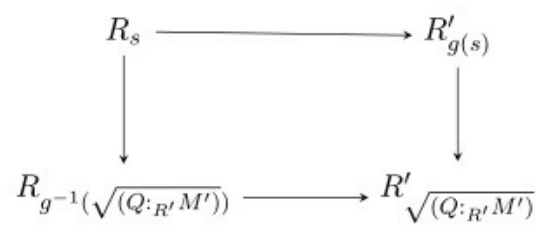

is a commutative diagram of natural maps, we define

$$
f^{\sharp}(U): \mathbb{O}_{X(M)}(U) \rightarrow f_{*} \mathbb{O}_{x\left(M^{\prime}\right)}(\mathcal{U})=\mathbb{O}_{X\left(M^{\prime}\right)}\left(f^{-1}(U)\right)
$$

which is given by $f^{\sharp}(U)(r)(Q)=g \sqrt{\left(Q:_{R^{\prime}} M^{\prime}\right)}(r(f(Q)))$ for each $r \in \mathbb{O}_{X(M)}(\mathcal{U})$ and $Q \in f^{-1}(\mathcal{U})$. Suppose $\mathcal{V} \subseteq \mathcal{U}$ and $Q \in f^{-1}(\mathcal{V})$. According to the following commutative diagram

We have $\left.g_{\sqrt{\left(Q:_{R^{\prime}} M^{\prime}\right)}} \circ r\right|_{\mathcal{V}} \circ f(Q)=\left.\left(g_{\sqrt{\left(Q:_{R^{\prime}} M^{\prime}\right)}} \circ r \circ f\right)\right|_{f^{-1}(\mathcal{V})}(Q)$. Considering the diagram

It is easy to check that

$$
\begin{gathered}
\rho_{f^{-1}(\mathcal{U}), f^{-1}(\mathcal{V})}^{\prime} f^{\sharp}(\mathcal{U})(r)(Q)=\rho_{f^{-1}(\mathcal{U}), f^{-1}(\mathcal{V})}^{\prime}{ }^{\sqrt{\left(Q:_{R^{\prime}} M^{\prime}\right)}} r \circ f(Q)= \\
\left.\left(g \sqrt{{ }_{\left(:_{R^{\prime}} M^{\prime}\right)}} r \circ f\right)\right|_{f^{-1}(\mathcal{V})}(Q)=\left.g \sqrt{\left(Q:_{\left.R^{\prime} M^{\prime}\right)}\right.} r\right|_{\mathcal{V}} \circ f(Q)=f^{\sharp}(\mathcal{V})(r \mid \mathcal{V})(Q)= \\
f^{\sharp}(\mathcal{V}) \rho u, \mathcal{V}(r)(Q) .
\end{gathered}
$$




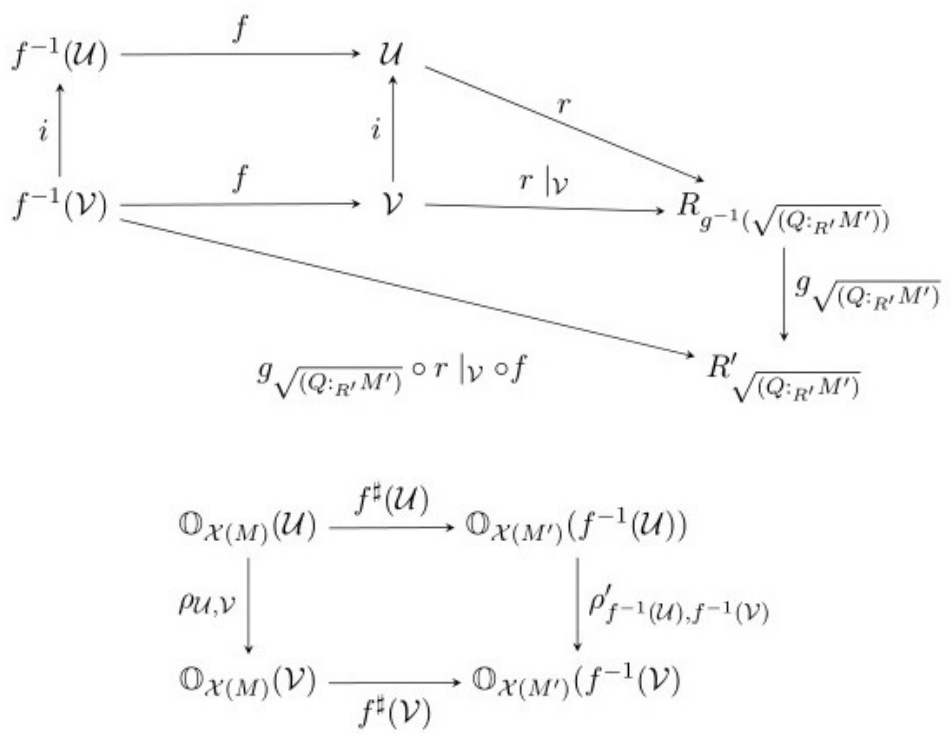

Thus the diagram is commutative and it follows that $f^{\sharp}: \mathbb{O}_{X(M)} \rightarrow f_{*} \mathbb{O}_{X\left(M^{\prime}\right)}$ is a morphism of sheaves. By Lemma 6, the map $f_{Q}^{\#}: \mathbb{O}_{X(M), f(Q)} \rightarrow \mathbb{O}_{X\left(M^{\prime}\right), Q}$ on stalks is clearly $R_{h\left(\sqrt{\left.\left(Q:_{R^{\prime}} M^{\prime}\right)\right)}\right.} \rightarrow R_{\sqrt{\left(Q:_{R^{\prime}} M^{\prime}\right)}}$. Thus the proof is completed.

Theorem 4. Let $s \in R$ and $M$ be a faithful $\phi$-module over a ring $R$. Then $\mathbb{O}_{\mathcal{X}(M)}\left(\mathcal{X}_{s}\right) \cong R_{s}$.

Proof. Suppose $\mu: R_{S} \rightarrow \mathbb{O}_{\mathcal{X}(M)}\left(\mathcal{X}_{s}\right)$ given by $\frac{a}{s^{n}} \mapsto\left(r: Q \mapsto \frac{a}{s^{n}} \in R_{\sqrt{(Q: M)}}\right)$. Indeed $\mu$ sends that $\frac{a}{s^{n}}$ to the section $r \in \mathbb{O}_{X(M)}\left(\mathcal{X}_{s}\right)$ which assigns to each $Q$ the image of $\frac{a}{s^{n}} \in R \sqrt{(Q: M)}$. It is clear that $\mu\left(\frac{a}{s^{n}}\right)$ is unique, since the range of $r$ is $\frac{a}{s^{n}}$. Therefore to show that $\mu$ is well-defined, it suffices to verify that $s^{n} \notin \sqrt{(Q: M)}$. Since $Q \in \mathcal{X}_{s}=\mathcal{X}(M)-v(s M)$, we have $\sqrt{(s M: M)} \nsubseteq \sqrt{(Q: M)}$. Now if $s^{n} \in$ $\sqrt{(Q: M)}$ (or equivalently $s \in \sqrt{(Q: M)}$ ), we have

$$
\begin{aligned}
r \in \sqrt{(s M: M)} & \Rightarrow r^{n} M \subseteq s M \subseteq \sqrt{(Q: M)} M \text { for some } n>0 \\
& \Rightarrow r^{n} \in(\sqrt{(Q: M)} M: M)=((\operatorname{rad}(Q): M) M: M) \\
& \Rightarrow r^{n} \in(\operatorname{rad}(Q): M)=\sqrt{(Q: M)} \\
& \Rightarrow r \in \sqrt{(Q: M)}
\end{aligned}
$$

which gives the contradiction $\sqrt{(s M: M)} \subseteq \sqrt{(Q: M)}$. Moreover $\mu$ is a homomorphism, since $\mathbb{O}_{X(M)}\left(\mathcal{X}_{s}\right)$ is a ring with the operations $\left(r_{1}+r_{2}\right)(Q)=r_{1}(Q)+$ $r_{2}(Q)$ and $\left(r_{1} r_{2}\right)(Q)=r_{1}(Q) r_{2}(Q)$. Now we are going to show that $\mu$ is injective. Let $\mu\left(\frac{a}{s^{n}}\right)=\mu\left(\frac{a^{\prime}}{s^{m}}\right)$, then for every $Q \in \mathcal{X}_{s}, \frac{a}{s^{n}}$ and $\frac{a^{\prime}}{s^{m}}$ have the same image in 
$R_{p}$, where $p=\sqrt{(Q: M)}$. Thus there exists $t \in R \backslash p$ such that $t\left(s^{m} a-s^{n} a^{\prime}\right)=0$. Let $I=\operatorname{Ann} n\left(s^{m} a-s^{n} a^{\prime}\right)$. Then $t \in I$ and $t \notin p$, so $I \nsubseteq p$. This happens for any $Q \in \mathcal{X}_{s}$. Hence we conclude that $V(I) \cap\left\{\sqrt{(Q: M)} \mid Q \in \mathcal{X}_{s}\right\}=\varnothing$ and so $\left\{\sqrt{(Q: M)} \mid Q \in \mathcal{X}_{f}\right\} \subseteq \operatorname{Spec}(R)-V(I)$. Since $M$ is a $\phi$-module, by Lemma 2 we have

$$
D_{s}=\left\{\sqrt{(Q: M)} \mid Q \in \mathcal{X}_{s}\right\} \subseteq D(I) .
$$

Therefore $s \in \sqrt{I}$ and so $s^{l} \in I$ for some positive integer $l$. Now we have $s^{l}\left(s^{m} a-\right.$ $\left.s^{n} a^{\prime}\right)=0$ which shows that $\frac{a}{s^{n}}=\frac{a^{\prime}}{s^{m}}$ in $R_{p}$. Thus $\mu$ is injective. Now we show that $\mu$ surjective. Assume $r \in \mathbb{O}_{\mathcal{X}(M)}\left(\mathcal{X}_{s}\right)$. Then we can cover $\mathcal{X}_{s}$ with open subset $\mathcal{V}_{i}$, on which $s$ is represented by $\frac{a_{i}}{b_{i}}$, with $b_{i} \notin \sqrt{(Q: M)}$ for all $Q \in \mathcal{V}_{i}$ and so $\mathcal{V}_{i} \subseteq \mathcal{X}_{b_{i}}$. By Lemma 5 , the open sets of the form $\mathcal{X}_{k}$ form a basis for the Zariski topology. So, we may assume that $\mathcal{V}_{i}=\mathcal{X}_{k_{i}}$ for some $k_{i} \in R$. Since $\mathcal{X}_{k_{i}} \subseteq \mathcal{X}_{b_{i}}$, by Lemma 4, $k_{i} \in \sqrt{R b_{i}}$. Thus $k_{i}^{n} \in R b_{i}$ for some $n \in \mathbb{N}$. So $k_{i}^{n}=c b_{i}$ and $\frac{a_{i}}{b_{i}}=\frac{c a_{i}}{c b_{i}}=\frac{c a_{i}}{k_{i}^{n}}$. We see that $r$ is represented by $\frac{a_{i}^{\prime}}{h_{i}},\left(a_{i}^{\prime}=c a_{i}, h_{i}=k_{i}^{n}\right)$ on $\mathcal{X}_{h_{i}}$ and (since $\left.\mathcal{X}_{k_{i}}=\mathcal{X}_{k_{i}^{n}}\right)$ the $\mathcal{X}_{h_{i}}$ cover $\mathcal{X}_{s}$. The open cover $\mathcal{X}_{s}=\cup \mathcal{X}_{h_{i}}$ has a finite subcover by Theorem 3. Assume $\mathcal{X}_{s} \subseteq \mathcal{X}_{h_{1}} \cup \cdots \cup \mathcal{X}_{h_{n}}$. For $1 \leq i, j \leq n$, $\frac{a_{i}^{\prime}}{h_{i}}$ and $\frac{a_{j}^{\prime}}{h_{j}}$ both represent $r$ on $X_{h_{i}} \cap X_{h_{j}}$. By Lemma $3 X_{h_{i}} \cap X_{h_{j}}=X_{h_{i} h_{j}}$ and by injectivity of $\mu$, we get $\frac{a_{i}^{\prime}}{h_{i}}=\frac{a_{j}^{\prime}}{h_{j}}$ in $R_{h_{i}} h_{j}$. Hence for some $n_{i j}$, we have $\left(h_{i} h_{j}\right)^{n_{i j}}\left(h_{j} a_{i}^{\prime}-h_{i} a_{j}^{\prime}\right)=0$. Let $m=\max \left\{n_{i j} \mid 1 \leq i, j \leq n\right\}$. Then

$$
h_{j}^{m+1}\left(h_{i} a_{i}^{\prime}\right)-h_{i}^{m+1}\left(h_{j} a_{j}^{\prime}\right)=0 .
$$

By replacing each $h_{i}$ by $h_{i}^{m+1}$, and $a_{i}^{\prime}$ by $h_{i} a_{i}^{\prime}$, we still see that $r$ is represented on $X_{h_{i}}$ by $\frac{a_{i}^{\prime}}{h_{i}}$, and furthermore, we have $h_{j} a_{i}^{\prime}=h_{i} a_{j}^{\prime}$ for all $i, j$. Since $\mathcal{X}_{s} \subseteq \mathcal{X}_{h_{1}} \cup$ $\cdots \cup \mathcal{X}_{h_{n}}$, by Lemma 2 we have

$$
D_{s}=\phi\left(\mathcal{X}_{s}\right) \subseteq \cup_{i=1}^{n} \phi\left(\mathcal{X}_{h_{i}}\right)=\cup_{i=1}^{n} D_{h_{i}} .
$$

Hence there are $c_{1}, \cdots, c_{n} \in R$ and $n^{\prime} \in \mathbb{N}$, such that $s^{n^{\prime}}=\sum_{i} c_{i} h_{i}$. Let $a=\sum_{i} c_{i} a_{i}^{\prime}$. Then for each $j$ we have

$$
h_{j} a=\sum_{i} c_{i} a_{i}^{\prime} h_{j}=\sum_{i} c_{i} h_{i} a_{j}^{\prime}=a_{j}^{\prime} s^{n^{\prime}} .
$$

It follows that $\frac{a}{s^{n^{\prime}}}=\frac{a_{j}^{\prime}}{h_{j}}$ on $\mathcal{X}_{h_{j}}$. So $\mu\left(\frac{a}{s^{n^{\prime}}}\right)=r$ everywhere, which shows that $\mu$ is surjective.

Corollary 5. Let $M$ be a faithful $\phi$-module over a ring $R$. Then $\mathbb{O}_{\mathcal{X}(M)}(\mathcal{X}(M)) \cong$ $R$.

Proof. Use Theorem 4. 
An affine scheme is a locally ringed space isomorphic as a locally ringed space to $\operatorname{Spec}(R)$ for some ring $R$. A scheme is a locally ringed space with the property that every point has an open neighborhood which is an affine scheme. A scheme is locally Noetherian if it can be covered by open affine subsets $\operatorname{Spec}\left(R_{i}\right)$, where each $R_{i}$ is a Noetherian ring. A scheme is Noetherian if it is locally Noetherian and quasi-compact [5].

Theorem 5. Let $M$ be a $\phi$-module over a ring $R$ such that $X(M)$ is a $T_{0^{-}}$ space. Then $\left(\mathcal{X}(M), \mathbb{O}_{X(M)}\right)$ is a scheme. Moreover, if $R$ is Noetherian, then $\left(X(M), \mathbb{O}_{X(M)}\right)$ is a Noetherian scheme.

Proof. Suppose $r \in R$. Therefore by Proposition $1, \phi \mid x_{r}$ is continuous. Also by Theorem 2, $\phi \mid \mathcal{X}_{r}$ is a bijection. Let $\mathscr{F}$ be a closed subset of $\mathcal{X}_{r}$. Then $\mathscr{F}=$ $\mathcal{X}_{r} \cap \nu(N)$ for some submodule $N$ of $M$. Hence $\phi(\mathcal{F})=\phi\left(\mathcal{X}_{r}\right) \cap V(\sqrt{(N: M)})$ is a closed subset of $\phi\left(\mathcal{X}_{r}\right)$. Thus $\phi \mid \mathcal{X}_{r}$ is a homeomorphism. Assume that $\mathcal{X}(M)=$ $\cup_{i \in I} \mathcal{X}_{r_{i}}$. Since $\phi$ is a bijection, then for $i \in I$ we have $\mathcal{X}_{r_{i}} \cong \phi\left(\mathcal{X}_{r_{i}}\right)=\{\sqrt{(Q: M)} \mid$ $\left.Q \in \mathcal{X}_{r_{i}}\right\}=D_{r_{i}} \cong \operatorname{Spec}\left(R_{r_{i}}\right)$. Thus by Theorem $4, \mathcal{X}_{r_{i}}$ is an affine scheme. So it implies that $\left(\mathcal{X}(M), \mathbb{O}_{\mathcal{X}(M)}\right)$ is a scheme. For the last statement, since $R$ is Noetherian, so is $R_{r_{i}}$ for each $i \in I$. Hence $\left(\mathcal{X}(M), \mathbb{O}_{\mathcal{X}(M)}\right)$ is a locally Noetherian scheme. By Corollary 3, $\mathcal{X}(M)$ is quasi-compact. Thus $\left(\mathcal{X}(M), \mathbb{O}_{\mathcal{X}(M)}\right)$ is a Noetherian scheme.

\section{ACKNOWLEDGEMENT}

We would like to thank the referee for a careful reading of our article and useful comments.

\section{REFERENCES}

[1] H. Ansari-Toroghy and S. Habibia, "The Zariski topology-graph of modules over commutative rings." Comm. Algebra, vol. 42, pp. 3283-3296, 2014, doi: 10.1080/00927872.2013.780065.

[2] H. Ansari-Toroghy and H. Ovlyaee-Sarmazdeh, "On the prime spectrum of a module and Zariski topologies." Comm. Algebra, vol. 38, pp. 4461-4475, 2010, doi: 10.1080/00927872.2013.780065.

[3] M. F. Atiyah and I. G. McDonald, Introduction to commutative algebra. Addison Weisley Publishing Company: Springer, 1969.

[4] H. Fazaeli Moghimi and F. Rashedi, "Primary-like submodules satisfying the primeful property." Transactions on Algebra and its Applications, vol. 1, no. 1, pp. 43-54, 2015.

[5] R. Hartshorne, Algebraic geometry. Graduate Text in Mathematics 52: Springer-Verlag, New York Inc, 1977.

[6] D. Hassanzadeh-Lelekaami and H. Roshan-Shekalgourabi, "Prime submodules and a sheaf on the prime spectra of modules." Comm. Algebra, vol. 42, pp. 3063-3077, 2014, doi: 10.1080/00927872.2013.780063.

[7] C. P. Lu, " Prime submodules of modules." Comment. Math. Univ. St. Paul, vol. 33, pp. 61-69, 1984.

[8] C. P. Lu, “ M-radical of submodules in modules.” Math. Japonica, vol. 34, pp. 211-219, 1989. 
[9] C. P. Lu, " The Zariski topology on the prime spectrum of a module." Houston J. Math., vol. 25, pp. 417-425, 1999.

[10] C. P. Lu, " A module whose prime spectrum has the surjective natural map." Houston J. Math., vol. 33, pp. 125-143, 2007.

[11] R. L. McCasland and M. E. Moore, “ Prime submodules.” Comm. Algebra, vol. 20, pp. 18031817, 1992, doi: 10.1080/00927879208824432.

[12] U. Tekir, "On the sheaf of modules." Comm. Algebra, vol. 33, pp. 2557-2562, 2005, doi: 10.1081/AGB-200065136.

Authors' addresses

H. Fazaeli Moghimi

University of Birjand, Department of Mathematics, P.O. Box 97175-615, Birjand, Iran

E-mail address: hfazaeli@birjand.ac.ir

F. Rashedi

University of Birjand, Department of Mathematics, P.O. Box 97175-615, Birjand, Iran

E-mail address: fatemehrashedi@birjand.ac.ir 